Preprint typeset in JHEP style. - HYPER VERSION

hep-th/0007235

\title{
Tachyon condensation in the D0/D4 system
}

\author{
Justin R. David \\ Department of Physics, University of California \\ Santa Barbara, CA 93106, USA. \\ justin@vulcan.physics.ucsb.edu
}

\begin{abstract}
The D0/D4 system with a Neveu-Schwarz B-field in the spatial directions of the D4-brane has a tachyon in the spectrum of the $(0,4)$ strings. The tachyon signals the instability of the system to form a bound state of the D0-brane with the D4-brane. We use the Wess-Zumino-Witten like open superstring field theory formulated by Berkovits to study the tachyon potential for this system. The tachyon potential lies outside the universality class of the D-brane anti-D-brane system. It is a function of the B-field. We calculate the tachyon potential at the zeroth level approximation. The minimum of the tachyon potential in this case is expected to reproduce the mass defect involved in the formation of the D0/D4 bound state. We compare the minimum of the tachyon potential with the mass defect in three cases. For small values of the B-field we obtain $70 \%$ of the expected mass defect. For large values of the B-field with $\operatorname{Pf}\left(2 \pi \alpha^{\prime} B\right)>0$ the potential reduces to that of the D-brane anti-D-brane reproducing $62 \%$ of the expected mass defect. For large values of the B-field with $\operatorname{Pf}\left(2 \pi \alpha^{\prime} B\right)<0$ the minimum of the tachyon potential gives $25 \%$ of the expected mass defect. At the tachyon condensate we show that the $(0,4)$ strings decouple from the low energy dynamics.
\end{abstract}

Keywords: D-branes, Tachyon condensation, Superstring field theory. 


\section{Contents}

1. Introduction 1

2. The Do/D4 system 3

3. String field theory of the $(0,4)$ strings

3.1 Open Superstring field theory 6

3.2 The tachyon vertex operator 7

3.3 The tachyon potential 8

4. Analysis of the tachyon potential 9

4.1 The calculation of the mass defect 9

4.2 The minimum of the tachyon potential and the mass defect 10

5. Excitations around the tachyon condensate

5.1 The decoupling of the $(0,4)$ strings 12

6. Conclusions 13

A. The twist operator and its correlation function 14

B. Details on the calculation of the tachyon potential 18

B.1 The quadratic term 18

B.2 The cubic term

B.3 The quartic term 19

\section{Introduction}

The presence of a tachyon in the spectrum of a string theory does not necessarily imply that the theory is inconsistent. In recent times there is accumulating evidence that the tachyon found in certain open string theories actually condense to a new stationary point of the potential. For example, the tachyon of the bosonic open string theory represents the instability of the D25-brane to decay to the vacuum. The tachyon of a non-BPS brane of Type II string theories also represents the instability of the non-BPS brane to decay to the vacuum.

Recent works have shown the existence of a stationary point in the tachyon potential. This potential is computed computed in string field theory using level 
truncation as an approximation method [1]. The value of the tachyon potential at this stationary point agrees with the tension of the unstable branes. This has been shown to a remarkable degree of accuracy both for the bosonic open string field theory and for the superstring field theory on the non-BPS brane in Type II string theories [2, 3, 4, 5, 7, 8, 9]. The physics of the solitons in the tachyon potential also seem to be reproduced in the level truncation approximation scheme [10, 11, 12]. Tachyon condensation has recently been studied in effective field theories on noncommutative spaces in [13, 14, 15, 16, 17] and its relationship to string field theory has been discussed in [18].

Tachyons are present in other unstable systems. They can be interpreted as the instability of the system to decay to stable systems. For all the cases analyzed so far in string field theory, the system decays to the vacuum. It would be interesting to find systems with tachyons, which decay to BPS states. For example consider the tachyon present for the open strings stretching between Dp-brane and $\mathrm{D}(\mathrm{p}+2)$ branes. This tachyonic potential was studied in first quantized string theory in [20]. It represented the instability of the system to for the system to form a bound state of $\mathrm{Dp}$ and $\mathrm{D}(\mathrm{p}+2)$-branes. Another reason to study other systems with tachyon is that one might be able to find tuning parameter in the tachyon potential. For the systems studied so far, the tachyon potential is universal [19]. Thus there is no tuning parameter which can be used to compare against the level truncation approximation scheme. In this paper we study a system with a tachyon which decays to a BPS state other than the vacuum. There is also a tuning parameter in the tachyon potential.

We consider the system of a single D0 and D4-brane with a Neveu-Schwarz Bfield in the spatial directions of the $\mathrm{D} 4$-brane. For generic values of the B-field there is a tachyon in the spectrum of the strings joining the D0-brane and the D4-brane [22]. The tachyon in the spectrum of the $(0,4)$ strings represents the instability of this system to form a bound state of D0 and D4-brane. The tachyon is in the NeveuSchwarz sector. We construct the tachyon vertex operator and use the Berkovits formalism of open superstring field theory to evaluate the tachyon potential at the zeroth level. The tachyon vertex contains operators involving matter primaries. Thus the potential falls outside the usual universality class of unstable branes in superstring theory. We show that there is a stationary point in the tachyon potential which approximates the mass defect in the formation of the D0/D4 bound state. The tachyon is almost on shell for small values of the B-field. Then the zeroth level approximation for the tachyon potential contribute to $70 \%$ of the mass defect. For large values of the B-field with with the Paffian, $\operatorname{Pf}\left(2 \pi \alpha^{\prime} B\right)>0$ we find that the tachyon potential reduces to that of a D0-brane anti-D0-brane pair. Thus reproducing $62 \%$ of the mass defect. For large of the B-field with $\operatorname{Pf}\left(2 \pi \alpha^{\prime} B\right)<0$ we find the zeroth level approximation contributes to $25 \%$ of the mass defect. The reason for this might be due to the presence of a large number of low lying states in the spectrum of the $(0,4)$ strings for this case [22]. 
The unstable D-brane decays to the closed string vacuum. Thus at the tachyon condensate the open string modes are not dynamical. We show that the corresponding statement for the D0/D4 bound state is that the $(0,4)$ strings decouple from the dynamics. To do this we look at the small fluctuations around the tachyon condensate. This is facilitated by the description of the D0/D4 bound state as an instanton in the noncommutative $U(1)$ gauge theory of the D4-brane. The tachyon instability is the instablity of zero size instanton in the noncommutative $U(1)$ gauge theory of the D4-brane. At the tachyon condensate the $(0,4)$ strings become massive and decouple from the dynamics. This is seen from the fact that the $(0,4)$ strings correspond to the scale of the instanton via the ADHM construction. The noncommutative $U(1)$ instanton does not have scale moduli. Thus at the level of the moduli space approximation we can see that the $(0,4)$ strings decouple from the dynamics at the tachyon condensate.

The organization of this paper is as follows. In Section 2 we review the D0/D4 system with the B-field in the spatial directions of the D4-brane. In Section 3 we set up the string field theory of the $(0,4)$ strings We write down the vertex operators corresponding to the tachyon and calculate the tachyon potential. In Section 4 we calculate the expected mass defect in the formation of the D0/D4 bound state and compare it with the value of the stationary point in the tachyon potential. In Section 5 we identify the tachyon condensate as the instanton of the noncommutative gauge theory on the D4-brane and show the decoupling of the $(0,4)$ strings. Section 6 contains our conclusions. Appendix A contains the correlation function of twist operators. Appendix B contains details on the calculation of the tachyon potential.

\section{The D0/D4 system}

In this section we review the D0/D4 system with a Neveu Schwarz B-field along the spatial direction of the D4-brane [22]. We discuss the mode expansions of the strings which join the D0-branes and the D4-branes.

Consider a single D0-brane and a single D4-brane of type IIA string theory in ten dimensions arranged as follows. The D4-brane is extended along the directions $x^{6}, x^{7}, x^{8}, x^{9}$. The open string spectrum consists of excitations of strings joining the D0-branes and the D4-branes among themselves and the excitations of open strings joining the D0-brane and the D4-brane. We denote the open strings joining the D0brane and the D4-brane as the $(0,4)$ strings. Let the string world sheet co-ordinates of the $(0,4)$ string be $X^{\mu}\left(\sigma^{0}, \sigma^{1}\right) . \mu$ runs from $0, \ldots 9$, and $\sigma^{1}$ lies between 0 and $\pi$. We work with Euclidean world sheet signature. Now turn on a constant B-field along the spatial directions of the D4-brane. We can choose a generic value of the 
B-field as given below

$$
B_{i j}=\frac{1}{2 \pi \alpha^{\prime}}\left(\begin{array}{cccc}
0 & b & 0 & 0 \\
-b & 0 & 0 & 0 \\
0 & 0 & 0 & b^{\prime} \\
0 & 0 & -b^{\prime} & 0
\end{array}\right)
$$

Where $i, j$ runs from $6 \ldots 9$. We choose the metric $g_{i j}=\delta_{i j}$. The boundary conditions of the world sheet co-ordinates with these moduli turned on is given by

$$
\begin{array}{r}
\partial_{\sigma^{1}} X^{i}+\left.2 \pi i \alpha^{\prime} B_{i j} \partial_{\sigma^{0}} X^{j}\right|_{\sigma^{1}=\pi}=0 \\
\left.\partial_{\sigma^{0}} X^{i}\right|_{\sigma^{1}=0}=0 \\
\left.\partial_{\sigma^{0}} X^{a}\right|_{\sigma^{1}=0, \sigma^{1}=\pi}=0 \text { where } a=1,2,3,4,5 \\
\left.\partial_{\sigma^{1}} X^{0}\right|_{\sigma^{1}=0, \sigma^{1}=\pi}=0
\end{array}
$$

The non-trivial mode expansions arise for the world sheet co-ordinates $X^{i}$, It is convenient to define co-ordinates

$$
\begin{array}{cl}
X^{+}=X^{6}+i X^{7} & X^{-}=X^{6}-i X^{7} \\
X^{+\prime}=X^{6}+i X^{7} & X^{-\prime}=X^{6}-i X^{7}
\end{array}
$$

The mode expansions of $X^{+}$and $X^{-}$are given by

$$
\begin{aligned}
& X^{+}=i \sqrt{\alpha^{\prime}} \sum_{n}\left[\frac{\alpha_{n-\nu}^{+}}{n-\nu} e^{-(n-\nu)\left(\sigma^{0}+i \sigma^{1}\right)}-\frac{\alpha_{n-\nu}^{+}}{n-\nu} e^{-(n-\nu)\left(\sigma^{0}-i \sigma^{1}\right)}\right] \\
& X^{-}=i \sqrt{\alpha^{\prime}} \sum_{n}\left[\frac{\alpha_{n+\nu}^{-}}{n+\nu} e^{-(n+\nu)\left(\sigma^{0}+i \sigma^{1}\right)}-\frac{\alpha_{n+\nu}^{-}}{n+\nu} e^{-(n+\nu)\left(\sigma^{0}-i \sigma^{1}\right)}\right]
\end{aligned}
$$

Where

$$
e^{2 \pi i \nu}=-\frac{1+i b}{1-i b}, \quad 0 \leq \nu<1
$$

The only non zero commutation relations are

$$
\left[\alpha_{n-\nu}^{+}, \alpha_{m+\nu}^{-}\right]=(n-\nu) \delta(n+m)
$$

Similar mode expansions arise for the world sheet co-ordinates $X^{+\prime}$ and $X^{-\prime}$. They are given by

$$
\begin{aligned}
& X^{+\prime}=i \sqrt{\alpha^{\prime}} \sum_{n}\left[\frac{\alpha_{n-\nu^{\prime}}^{+\prime}}{n-\nu^{\prime}} e^{-\left(n-\nu^{\prime}\right)\left(\sigma^{0}+i \sigma^{1}\right)}-\frac{\alpha_{n-\nu^{\prime}}^{+\prime}}{n-\nu^{\prime}} e^{-\left(n-\nu^{\prime}\right)\left(\sigma^{0}-i \sigma^{1}\right)}\right] \\
& X^{-\prime}=i \sqrt{\alpha^{\prime}} \sum_{n}\left[\frac{\alpha_{n+\nu^{\prime}}^{-\prime}}{n+\nu^{\prime}} e^{-\left(n+\nu^{\prime}\right)\left(\sigma^{0}+i \sigma^{1}\right)}-\frac{\alpha_{n+\nu^{\prime}}^{-\prime}}{n+\nu^{\prime}} e^{-\left(n+\nu^{\prime}\right)\left(\sigma^{0}-i \sigma^{1}\right)}\right]
\end{aligned}
$$

Where

$$
e^{2 \pi i \nu^{\prime}}=-\frac{1+i b^{\prime}}{1-i b^{\prime}} \quad 0 \leq \nu^{\prime}<1
$$


Similarly the only non zero commutation relations are

$$
\left[\alpha_{n-\nu^{\prime}}^{+\prime}, \alpha_{m+\nu^{\prime}}^{-\prime}\right]=\left(n-\nu^{\prime}\right) \delta(n+m)
$$

The mode expansions of world sheet superpartners of the bosonic fields are fixed by supersymmetry. The mode expansions of $\psi^{+}$and $\bar{\psi}^{+}$of $X^{+}$is given by

$$
\begin{aligned}
& \psi^{+}=-i \sqrt{\alpha^{\prime}} \sum_{n} \psi_{n+1 / 2-\nu}^{+} e^{-(n+1 / 2-\nu)\left(\sigma^{0}+i \sigma^{1}\right)} \\
& \bar{\psi}^{+}=i \sqrt{\alpha^{\prime}} \sum_{n} \psi_{n+1 / 2-\nu}^{+} e^{-(n+1 / 2-\nu)\left(\sigma^{0}-i \sigma^{1}\right)}
\end{aligned}
$$

The mode expansions of the the superpartners of $X^{-}$are given by

$$
\begin{aligned}
& \psi^{-}=-i \sqrt{\alpha^{\prime}} \sum_{n} \psi_{n+1 / 2+\nu}^{+} e^{-(n+1 / 2+\nu)\left(\sigma^{0}+i \sigma^{1}\right)} \\
& \bar{\psi}^{+}=i \sqrt{\alpha^{\prime}} \sum_{n} \psi_{n+1 / 2+\nu}^{+} e^{-(n+1 / 2+\nu)\left(\sigma^{0}-i \sigma^{1}\right)}
\end{aligned}
$$

We have written the mode expansions in the Neveu-Schwarz sector. The only nonzero anti-commutation rules are given by

$$
\left\{\psi_{n+1 / 2-\nu}^{+}, \psi_{m+1 / 2+\nu}^{-}\right\}=\delta(m+n)
$$

The mode expansions and the anti-commutation relations for the superpartners of $X^{\prime-}$ and $X^{\prime+}$ are given by replacing the variables in (2.10), (2.11) and (2.12) by their primed variables.

To show that the $(0,4)$ strings have a tachyon in their spectrum we evaluate the zero point energy. The zero point energy for four of the lowest energy states in the Neveu-Schwarz sector are given by

$$
\begin{aligned}
E_{-}^{+}=-\frac{1}{2}+\frac{1}{2}\left(\nu+\nu^{\prime}\right) & E_{+}^{+}=\frac{1}{2}-\frac{1}{2}\left(\nu+\nu^{\prime}\right) \\
E_{-}^{-}=\frac{\nu-\nu^{\prime}}{2} & E_{+}^{-}=\frac{\nu^{\prime}-\nu}{2}
\end{aligned}
$$

Out of these four states two of them are projected out by the GSO projection. For the case of the D0-brane and the D4-brane the states with zero point energies $E_{ \pm}^{+}$ are retained [22]. Thus we see unless $\nu+\nu^{\prime}=1$ there is a tachyon in the spectrum

of the $(0,4)$ strings. For $\nu+\nu^{\prime}<1$ the tachyon (mass) $)^{2}$ is given by $E_{-}^{+} /\left(2 \alpha^{\prime}\right)$ and for $\nu+\nu^{\prime}>1$ the tachyon (mass) $)^{2}$ is given by $E_{+}^{-} /\left(2 \alpha^{\prime}\right)$.

\section{String field theory of the $(0,4)$ strings}

In this section we will compute the potential for the tachyon in the spectrum of the $(0,4)$ strings using string field theory. We will compute the tachyon potential upto the zeroth level in the level truncation approximation. We have seen in section 2 that the tachyon is in the Neveu-Schwarz sector. Therefore we can use the Wess-ZuminoWitten like open superstring field theory formulated by Berkovits [21] to calculate the tachyon potential. 


\subsection{Open Superstring field theory}

We will briefly review open superstring field theory. From this section onwards we work in the units $\alpha^{\prime}=1$ The string field theory action is given by

$$
S=\frac{1}{g^{2}}\left\langle\left\langle\left(e^{-\Phi} Q_{B} e^{\Phi}\right)\left(e^{-\Phi} \eta_{0} e^{\Phi}\right)-\int_{0}^{1} d t\left(e^{-t \Phi} \partial_{t} e^{t \Phi}\right)\left\{\left(e^{-t \Phi} Q_{B} e^{t \Phi}\right),\left(e^{-t \Phi} \eta_{0} e^{t \Phi}\right)\right\}\right\rangle\right\rangle,
$$

where $\{A, B\} \equiv A B+B A$. The open string coupling constant of the $(0,4)$ strings, $g$ is related to the closed string coupling constant coupling constant $g_{c}$ by $g^{2}=g_{c}$. The presence of the Neveu-Schwarz B-field does not change the relationship of the open string coupling constant to the closed string coupling constant. This is because the B-field is perpendicular to the common Neumann directions of the $(0,4)$ strings. The string field $\hat{\Phi}$ for the $(0,4)$ strings is given by

$$
\hat{\Phi}=\Phi_{+}^{(1)} \otimes I \otimes I+\Phi_{+}^{(2)} \otimes \sigma_{3} \otimes I+\Phi_{-}^{(3)} \otimes \sigma_{1} \otimes \sigma_{1}+\Phi_{-}^{(4)} \otimes \sigma_{2} \otimes \sigma_{1}
$$

Where $\Phi_{+}^{(1)}, \Phi_{+}^{(2)}$ stand for GSO even open string vertex operators and $\Phi_{-}^{(3)}, \Phi_{-}^{(4)}$ stand for GSO odd open string vertex operators. These operators have ghost number 0 and picture number 0 in the combined conformal field theory of a $c=15$ superconformal matter system and the $b, c, \beta, \gamma$ ghost system with $c=-15$. The bosonized ghost fields $\xi, \eta, \phi$ are related to $\beta, \gamma$ by

$$
\beta=\partial \xi e^{-\phi}, \quad \gamma=\eta e^{\phi}
$$

$\sigma_{1}, \sigma_{2}, \sigma_{3}$ are $2 \times 2$ pauli matrices and I is the $2 \times 2$ identity matrix. The first set of matrices are the external Chan-Paton factors and the second set of matrices are the internal Chan-Paton factors. $\hat{Q}_{B}=Q_{B} \otimes I \otimes \sigma_{3}$ where $Q_{B}$ is given by

$$
\begin{aligned}
Q_{B} & =\oint d z j_{B}(z)=\oint d z\left\{c\left(T_{m}+T_{\xi \eta}+T_{\phi}+c \partial c b+\eta e^{\phi} G_{m}-\eta \partial \eta e^{2 \phi} b\right\}\right. \\
T_{\xi \eta} & =\partial \xi \eta \\
T_{\phi} & =-\frac{1}{2} \partial \phi \partial \phi-\partial^{2} \phi
\end{aligned}
$$

$T_{m}$ is the mater stress tensor and $G_{m}$ is the matter superconformal generator. $\hat{\eta}_{0}=$ $\eta_{0} \otimes I \otimes \sigma_{3}$ and $\eta_{0}$ is the zeroth mode of the field $\eta$. The products of operators in $\langle\langle\cdots\rangle\rangle$ is defined by

$$
\left\langle\left\langle A_{1} \ldots A_{n}\right\rangle\right\rangle=\left\langle f_{1}^{(n)} \circ A_{1}(0) \cdots f_{n}^{(n)} \circ A_{n}(0)\right\rangle
$$

Here $\langle\cdots\rangle$ denotes the correlation function evaluated on the disc including the trace over the internal and the ordinary (external) Chan-Paton factors. $f \circ A$ denotes the conformal transform of $A$ by $f$. For the case of the disc the functions $f_{k}^{(n)}$ are given by

$$
f_{k}^{(n)}(z)=e^{\frac{2 \pi i(k-1)}{n}}\left(\frac{1+i z}{1-i z}\right)^{2 / n} \text { for } n \geq 1
$$


We now expand (3.1) to compute the tachyon potential to the zeroth level. For this it is sufficient to retain only terms upto the fourth order in the string field in the expansion of (3.1). This is given by

$$
\begin{aligned}
S & =\frac{1}{2 g^{2}}\left\langle\left\langle\frac{1}{2}\left(\hat{Q}_{B} \Phi\right)\left(\hat{\eta}_{0} \hat{\Phi}\right)+\frac{1}{6}\left(\hat{Q}_{B} \hat{\Phi}\right)\left(\hat{\Phi}\left(\hat{\eta}_{0} \Phi\right)-\left(\hat{\eta}_{0} \hat{\Phi} \hat{\Phi}\right)\right)\right.\right. \\
& \left.\left.+\frac{1}{24}\left(\hat{Q}_{B} \hat{\Phi}\right)\left(\hat{\Phi}^{2}\left(\hat{\eta}_{0} \hat{\Phi}\right)-2 \hat{\Phi}\left(\hat{\eta}_{0} \hat{\Phi}\right)+\left(\hat{\eta}_{0} \hat{\Phi}\right) \hat{\Phi}^{2}\right)\right\rangle\right\rangle
\end{aligned}
$$

\subsection{The tachyon vertex operator}

We write down the vertex operator corresponding to the tachyon and the first excited state in the spectrum of the $(0,4)$ strings. We work in the gauge [6]

$$
b_{0} \hat{\Phi}=0, \quad \xi_{0} \hat{\Phi}=0
$$

Let us take without loss of generality $\nu+\nu^{\prime}<1$. Then the tachyon vertex operator is given by

$$
\hat{T}=\xi c e^{-\phi}\left(t_{+} \Sigma_{\nu} \Sigma_{\nu^{\prime}} \otimes \sigma_{+} \otimes \sigma_{1}+t_{-} \Sigma_{-\nu} \Sigma_{-\nu^{\prime}} \otimes \sigma_{-} \otimes \sigma_{1}\right)
$$

where

$$
\Sigma_{\nu} \Sigma_{\nu^{\prime}}=\sigma_{\nu} e^{i \nu H} \sigma_{\nu^{\prime}} e^{i \nu^{\prime} H} \quad \Sigma_{-\nu} \Sigma_{-\nu^{\prime}}=\sigma_{-\nu} e^{-i \nu H} \sigma_{-\nu^{\prime}} e^{-i \nu^{\prime} H}
$$

The twist operators $\sigma_{ \pm \nu}, \sigma_{ \pm \nu^{\prime}}$ and $H, H^{\prime}$ are defined in the appendix. $\sigma_{+}$and $\sigma_{-}$are given by

$$
\sigma_{+}=\left(\begin{array}{ll}
0 & 1 \\
0 & 0
\end{array}\right) \quad \sigma_{-}=\left(\begin{array}{ll}
0 & 0 \\
1 & 0
\end{array}\right)
$$

The first set of matrices in (3.9) stand for the external Chan-Paton indices, the second set stand for the internal Chan-Paton indices. The twist operator $\Sigma_{\nu} \Sigma_{\nu^{\prime}}$ and the anti-twist operator $\Sigma_{-\nu} \Sigma_{-\nu^{\prime}}$ comes with external Chan-Paton factors $\sigma_{+}$and $\sigma_{-}$ respectively. This is because the insertion of $\Sigma_{\nu} \Sigma_{-\nu}$ changes the boundary conditions to that of the string joining the D0-brane to the D4-brane. The mode expansion of these strings are given in (2.4), (2.7), (2.10), (2.11). While the insertion of $\Sigma_{-\nu} \Sigma_{-\nu^{\prime}}$ changes the boundary conditions to that of the string joining the D4-brane to the D0-brane, that is a string of opposite orientation. Note that the conformal dimension of the tachyon vertex agrees with the zero point energy $E_{-}^{+}$.

The tachyon vertex operator in (3.9) reduces to the tachyon vertex of a D-brane anti-D-brane pair when $\nu=0$ and $\nu^{\prime}=0$. When $\nu=0$ and $\nu^{\prime}=0$ we see from the mode expansions in (2.4), (2.7), (2.10), (2.11) the boundary condition is Dirichlet at both ends of the $(0,4)$ strings. From (2.5) and (2.8) we see that $\nu=0$ and $\nu^{\prime}=0$ can occur only if $b \rightarrow \infty$ and $b^{\prime} \rightarrow \infty$ or $b \rightarrow \infty$ and $b^{\prime} \rightarrow \infty$. The D0-brane charge induced on the D4-brane is proportional to $-\operatorname{Pf}(2 \pi B)=-b b^{\prime}$. The D4-brane

effectively becomes an anti-D0-brane. Thus the tachyon vertex in (3.9) reduces to the tachyon vertex of a D-brane anti-D-brane pair. 
For completeness we include the vertex operator of the first excited state. The first excited state becomes the tachyon and the tachyon in (3.9) becomes the first excited state when $\nu+\nu^{\prime}>1$. The vertex operator for the first excited state is given by

$$
\hat{E}=\xi c e^{-\phi}\left(e_{+} \Sigma_{1-\nu} \Sigma_{1-\nu^{\prime}} \otimes \sigma_{+} \otimes \sigma_{1}+e_{-} \Sigma_{-(1-\nu)} \Sigma_{-\left(1-\nu^{\prime}\right)} \otimes \sigma_{-} \otimes \sigma_{1}\right)
$$

where

$$
\begin{aligned}
\Sigma_{1-\nu} \Sigma_{1-\nu^{\prime}} & =\sigma_{1-\nu} e^{i(1-\nu) H} \sigma_{1-\nu^{\prime}} e^{i\left(1-\nu^{\prime}\right) H^{\prime}} \\
\Sigma_{-(1-\nu)} \Sigma_{-\left(1-\nu^{\prime}\right)} & =\sigma_{-(1-\nu)} e^{-i(1-\nu) H} \sigma_{-\left(1-\nu^{\prime}\right)} e^{-i\left(1-\nu^{\prime}\right) H^{\prime}}
\end{aligned}
$$

To compute the tachyon potential to the zeroth level we take the string field to be

$$
\hat{\Phi}=\hat{T}+\hat{E}
$$

\subsection{The tachyon potential}

In the case of the unstable brane or the D-brane anti-D-brane system the tachyon potential is universal [19]. The tachyon potential in this case is independent of the background conformal field theory except for an overall multiplicative factor. ${ }^{1}$ The computation of the tachyon potential for the case involves correlations functions involving the ghosts fields and the matter energy momentum tensor with central charge 15. It is clear from the vertex operator for the tachyon in (3.9) that it involves matter primaries. Thus the tachyon potential for the $(0,4)$ strings does not belong to the same universality class as that of the unstable D-branes. In fact it depends on the background B-field.

The details on the computation of the tachyon potential is given in appendix B. We state the result. The tachyon potential of the $(0,4)$ strings at the zeroth level is given by

$$
\begin{aligned}
V & =-S=\frac{1}{g^{2}}\left(-\frac{1}{2}\left(1-\left(\nu+\nu^{\prime}\right) t_{-} t_{+}++\frac{1}{2}\left(1-\left(\nu+\nu^{\prime}\right) e_{-} e_{+}\right.\right.\right. \\
& +\frac{1}{F(\nu, 1-\nu, 1 ; 1 / 2) F\left(\nu^{\prime}, 1-\nu^{\prime}, 1 ; 1 / 2\right)} t_{-}^{2} t_{+}^{2} \\
& \left.+\frac{e_{-}^{2} e_{+}^{2}}{F(1-\nu, \nu, 1 ; 1 / 2) F\left(1-\nu^{\prime}, \nu^{\prime}, 1 ; 1 / 2\right)}+\frac{2 t_{+} t_{-} e_{+} e_{-}}{F(\nu, 1-\nu, 1 ;-1) F\left(\nu^{\prime}, 1-\nu^{\prime}, 1 ;-1\right)}\right)
\end{aligned}
$$

where the hypergeometric function $F(\nu, 1-\nu, 1 ; x)$ is defined in (A.12). We have the relation 23]

$$
F(a, 1-a, 1 ; 1 / 2)=\sqrt{\pi} \frac{1}{\Gamma\left(\frac{1}{2}+\frac{a}{2}\right) \Gamma\left(1-\frac{a}{2}\right)}
$$

\footnotetext{
${ }^{1}$ The overall factor is the open string coupling constant. The relationship of the open string coupling constant to the closed string coupling constant can depend on the background conformal field theory.
} 
Using this, the tachyon potential becomes

$$
\begin{aligned}
V & =\frac{1}{g^{2}}\left(-\frac{1}{2}\left(1-\left(\nu+\nu^{\prime}\right)\right) t_{-} t_{+}+\frac{1}{2}\left(1-\left(\nu+\nu^{\prime}\right)\right) e_{-} e_{+}\right. \\
& +\frac{1}{\pi} \Gamma\left(\frac{1}{2}+\frac{\nu}{2}\right) \Gamma\left(1-\frac{\nu}{2}\right) \Gamma\left(\frac{1}{2}+\frac{\nu^{\prime}}{2}\right) \Gamma\left(1-\frac{\nu^{\prime}}{2}\right)\left(t_{-}^{2} t_{+}^{2}+e_{-}^{2} e_{+}^{2}\right) \\
& \left.+\frac{2 t_{+} t_{-} e_{+} e_{-}}{F(\nu, 1-\nu, 1 ;-1) F\left(\nu^{\prime}, 1-\nu^{\prime}, 1 ;-1\right)}\right)
\end{aligned}
$$

\section{Analysis of the tachyon potential}

The mass defect for D-brane anti-D-brane annihilation is given by $-2 \times$ (mass of the D-brane). There is a minimum of the tachyon potential computed by the level truncation approximation scheme which approximates the mass defect. For the D0/D4 system with the Neveu-Schwarz B-field in the spatial direction we expect the tachyon condensate to be the bound state of the D0-brane within the D4-brane. Thus we expect the minimum of the tachyon potential of the $(0,4)$ strings to approximate the mass defect of the formation of the D0/D4 bound state from the D0-brane and the D4-brane. In this section we will compare this mass defect with the value of the minimum of the tachyon potential.

\subsection{The calculation of the mass defect}

The mass of the D0-brane in the conventions of [19, 6] is given by

$$
M_{D 0}=\frac{1}{2 \pi^{2} g^{2}}
$$

This formula is true for any Dp-brane. In calculating this mass the longitudinal directions of the Dp-brane is assumed to be compact. The mass of the D4-brane with the B-field in the spatial directions is given by

$$
M_{D 4}=\frac{1}{2 \pi^{2} g^{2}} \sqrt{\left(1+b^{2}\right)\left(1+b^{\prime 2}\right)}
$$

We can understand this expression from the Dirac-Born-Infeld action.

$$
S=\frac{1}{2 \pi^{2} g^{2}} \sqrt{\operatorname{Det}(G+2 \pi B)}
$$

where $G$ is the induced metric. Substituting the value of the B-field in (2.1) and the metric $g_{i j}=\delta_{i j}$ and expanding the Dirac-Born Infeld action in the static gauge we obtain the mass for the D4-brane with the B-field as given in (4.2). It is instructive to understand the formula in (4.2) by another method. The BPS formula for the D0/D4 system with moduli is given by [24]

$$
M^{2}=\left(Q_{0}+(1-\operatorname{Pf}(2 \pi B)) Q_{4}\right)^{2}+\left(2 \pi^{2} B_{i k} g^{i j} g^{j l} B_{k l}+2 \operatorname{Pf}(2 \pi B)\right) Q_{4}^{2}
$$


Here $Q_{0}=N_{0} /\left(2 \pi^{2} g^{2}\right)$ and $Q_{4}=N_{4} /\left(2 \pi^{2} g^{2}\right) . \quad N_{0}$ and $N_{4}$ are the number of D0branes and D4-branes respectively. Substituting the values of the B-field from (2.1) we get

$$
M^{2}=\left(Q_{0}+\left(1-b b^{\prime}\right) Q_{4}\right)^{2}+\left(b+b^{\prime}\right)^{2} Q_{4}^{2}
$$

Substituting $Q_{0}=0$ and $Q_{4}=1 /\left(2 \pi^{2} g^{2}\right)$ in (4.5) we get (4.2).

Now it is easy to write down the mass formula for the D0/D4 bound state. Substitute $Q_{0}=1 /\left(2 \pi^{2} g^{2}\right)$ and $Q_{4}=1 /\left(2 \pi^{2} g^{2}\right)$ in (4.5) we get the following mass formula for the D0/D4 bound state.

$$
\begin{aligned}
M_{D 0 / D 4} & =\frac{1}{2 \pi^{2} g^{2}} \sqrt{\left(1+\left(1-b b^{\prime}\right)\right)^{2}+\left(b+b^{\prime}\right)^{2}} \\
& =\frac{1}{2 \pi^{2} g^{2}} \sqrt{4+b^{2}+b^{\prime 2}-2 b b^{\prime}+b^{2} b^{\prime 2}}
\end{aligned}
$$

Thus the mass defect is given by

$$
\Delta M=M_{D 0 / D 4}-\left(M_{D 0}+M_{D 4}\right)
$$

We analyze the mass defect $\Delta M$ in three limiting cases.

Case 1. $b \ll 1, b^{\prime} \ll 1$

The leading order terms in the mass defect is given by

$$
\Delta M_{1}=-\frac{1}{2 \pi^{2} g^{2}}\left(\frac{b+b^{\prime}}{2}\right)^{2}
$$

Case 2. $b \rightarrow \infty, b^{\prime} \rightarrow \infty$ or $b \rightarrow-\infty, b^{\prime} \rightarrow-\infty ; \operatorname{Pf}(2 \pi B)>0$

The leading order terms in the mass defect is given by

$$
\Delta M_{2}=-2 \frac{1}{2 \pi^{2} g^{2}}
$$

Notice that in this case the mass defect reduces to that of the D-brane anti-D-brane system. This is expected from the discussion in Section 3.2

Case 3. $b \rightarrow \infty, b^{\prime} \rightarrow-\infty$ or $b \rightarrow-\infty, b^{\prime} \rightarrow \infty ; \operatorname{Pf}(2 \pi B)<0$

For this case the leading order terms in the mass defect is given by

$$
\Delta M_{3}=-\frac{1}{4 \pi^{2} g^{2}}\left(\frac{1}{b}+\frac{1}{b^{\prime}}\right)^{2}
$$

\subsection{The minimum of the tachyon potential and the mass defect}

In this section we compute the minimum of the tachyon potential and compare it with the mass defect obtained in section 4.1. The tachyon potential in (3.17) has an extrema at

$$
\begin{array}{ll}
t_{-} t_{+}=\frac{\pi}{4} \frac{1-\left(\nu+\nu^{\prime}\right)}{\Gamma(1 / 2+\nu / 2) \Gamma(1-\nu / 2) \Gamma\left(1 / 2+\nu^{\prime} / 2\right) \Gamma\left(1-\nu^{\prime} / 2\right)} \\
e_{-}=0 & e_{+}=0
\end{array}
$$


The minimum of the tachyon potential at this extrema is given by

$$
V_{\min }=-\frac{\pi}{16 g^{2}} \frac{\left(1-\left(\nu+\nu^{\prime}\right)\right)^{2}}{\Gamma(1 / 2+\nu / 2) \Gamma(1-\nu / 2) \Gamma\left(1 / 2+\nu^{\prime} / 2\right) \Gamma\left(1-\nu^{\prime} / 2\right)}
$$

We now compare this to the mass defect obtained in the three cases in section 4.1

Case 1. $b \ll 1, b^{\prime} \ll 1$

To the leading order the value of $\nu$ and $\nu^{\prime}$ are given by

$$
\nu=\frac{1}{2}-\frac{b}{\pi} \quad \nu^{\prime}=\frac{1}{2}-\frac{b^{\prime}}{\pi}
$$

We substitute this values of $\nu$ and $\nu^{\prime}$ in (4.12). We find the minimum of the tachyon to the leading order is given by

$$
V_{\min }=-\frac{1}{2 g^{2} \pi^{2}}\left(\frac{b+b^{\prime}}{2}\right)^{2} \frac{\pi}{2(\Gamma(3 / 4))^{4}}
$$

On comparison with the mass defect for this case from (4.8) we obtain $70 \%$ of the expected result.

$$
\frac{V_{\min }}{\Delta M_{1}}=\frac{\pi}{2\left(\Gamma(3 / 4)^{4}\right.}=.70
$$

Case 2. $b \rightarrow \infty, b^{\prime} \rightarrow \infty$ or $b \rightarrow-\infty, b^{\prime} \rightarrow-\infty ; \operatorname{Pf}(2 \pi B)>0$

As we have seen in section 3.2 this case is expected to reduce that of the D0-brane anti-D0-brane system. Without loss of generality we take $b \rightarrow-\infty, b^{\prime} \rightarrow-\infty$. The value of $\nu$ and $\nu^{\prime}$ to the leading order are given by

$$
\nu=-\frac{1}{\pi b} \quad \nu^{\prime}=-\frac{1}{\pi b^{\prime}}
$$

It is easy to see the tachyon potential in (3.17) reduces to that of the D0-brane anti-D0-brane system. The minimum of the tachyon potential is given by

$$
V_{\min }=-\frac{1}{16 g^{2}}
$$

This is the value of the minimum of the tachyon potential computed in [5, 6] for the D-brane anti-D-brane system. Comparison with the mass defect in (4.9) gives $62 \%$ of the expected result.

$$
\frac{V_{\min }}{\Delta M_{2}}=\frac{\pi^{2}}{16}=.62
$$

Case 3. $b \rightarrow \infty, b^{\prime} \rightarrow-\infty$ or $b \rightarrow-\infty, b^{\prime} \rightarrow \infty ; \operatorname{Pf}(2 \pi B)<0$

Without loss of generality we can consider the case $b \rightarrow \infty, b^{\prime} \rightarrow-\infty$. For this case the values of $\nu$ and $\nu^{\prime}$ to the leading order are given by

$$
\nu=1-\frac{1}{\pi b} \quad \nu^{\prime}=-\frac{1}{\pi b^{\prime}}
$$


Substituting this in (4.12) we obtain

$$
V_{\min }=-\frac{1}{4 \pi^{2} g^{2}}\left(\frac{1}{b}+\frac{1}{b^{\prime}}\right)^{2} \frac{1}{4}
$$

Comparing the value of the minimum of the tachyon potential to the mass defect in (4.1) yields only $25 \%$ of the expected result.

$$
\frac{V_{\min }}{\Delta M_{2}}=\frac{1}{4}=.25
$$

Perhaps the low contribution from the zeroth level approximation to the tachyon potential might be due to the large number of low lying states found for this case [22].

It is nice to see that we obtain the same dependence of the mass defect on the B-field in Case 1 and Case 2 from two different functions. Graphically it can be easily seen that $V_{\min } \leq \Delta M$ for all values of $\nu$ and $\nu^{\prime}$ between 0 and 1 . Equality holds only when $\nu+\nu^{\prime}=1$, that is when the tachyon is massless and there is no mass defect. The best approximation to the mass defect over all the allowed values of $\nu$ and $\nu^{\prime}$ is $70 \%$.

\section{Excitations around the tachyon condensate}

In this section we examine the excitations of the $(0,4)$ strings around the D0/D4 bound state. This is facilitated by the description of the D0/D4 bound state from the gauge theory of the D4-brane. The existence of the bound state was shown from the gauge theory in 25]. The bound state of the D0-brane within the D4-brane can be identified with a single instanton of the D4-brane gauge theory. We will briefly review the arguments here.

The gauge theory of the D4-brane with the B-field is a noncommutative $U(1)$ gauge theory with $N=4$ supersymmetry. This theory admits non-singular instanton solutions [26]. This noncommutative $U(1)$ gauge theory with a single instanton has the right properties to be identified as the bound state the D0-brane within the D4brane. The arguments for this is the same as the case with no B-field. The instanton in the D4-brane gauge theory carries the Ramond-Ramond charge of the D0-brane. It preserves the same supercharges as the D0-brane [22]. The instanton of the D4brane gauge theory has the right action as the tension of the D0-brane. The ADHM construction of the instanton of noncommutative D4-brane gauge theory reproduces the moduli space of the D0-brane gauge theory [26].

\subsection{The decoupling of the $(0,4)$ strings}

In this section we show that at the tachyon condensate the $(0,4)$ strings become massive and decouple from the dynamics. 
We examine the small fluctuations around the tachyon condensate. The tachyon condensate is identified with the instanton of the non-commutative gauge theory. The small fluctuations around the instanton can be determined by examining the moduli space of the instanton of the noncommutative $U(1)$ gauge theory. This is given by the ADHM construction of the instanton. The moduli space is determined by solving the following equations

$$
\begin{aligned}
A B & =0 \\
|A|^{2}-|B|^{2} & =r
\end{aligned}
$$

These are the D-terms of a $(4,4)$ supersymmetric $U(1)$ gauge theory with one hypermultiplet, that is the gauge theory of the D0-brane. $r$ is the Fayet-Illiopoulous term. It has been shown that a bound state exists for $r>0$ [25]. For Case 1 and Case $2 r$ has been identified to be proportional to the square of the self dual part of the B-field [22]. $A$ and $B$ are the bosonic components of the hypermultiplet. $A$ is is a complex field which carries a charge of +1 under the $U(1)$ gauge symmetry and $B$ carries a charge of -1 under the $U(1)$ gauge symmetry. To determine the moduli space we must solve the equations in (5.1) and identify gauge equivalent configurations.

The solution of (5.1) is given by $B=0$ with $|A|=\sqrt{r}$. Using the $U(1)$ gauge degree of freedom one can choose the solution $B=0$ and $A=\sqrt{r}$. Thus we see there is no modulus for the non-commutative instanton other than its position.

Small fluctuations of the $(0,4)$ strings can be identified with the small fluctuations of the fields $A$ and $B$. This can be seen from the fact that the fields corresponding to the $(0,4)$ strings are hypermultiplets in the gauge theory of the D4-brane and the D0-brane. Therefore at the tachyon condensate, the $(0,4)$ strings are massive and decouple from the low energy dynamics. The decoupling of the $(0,4)$ strings is the analog of the decoupling of the strings joining the D-brane with the anti-D-brane. It is the analog of the decoupling of the relative $U(1)$ for the D-brane, anti-D-brane case.

\section{Conclusions}

We have studied tachyon condensation in the D0/D4 system with B-field in the spatial directions of the D4-brane using string field theory. The tachyon in the spectrum of the $(0,4)$ strings signals the instability of the D0/D4 system to form the D0/D4 bound state. Here after tachyon condensation we are left with a BPS state. We computed the tachyon potential in the zeroth level approximation. This tachyon potential is outside the universality class analyzed for the D-brane anti-Dbrane systems. It is a function of the background B-field. We compared the minimum of the tachyon potential to the mass defect for the formation of the D0/D4 bound state in three cases. When the tachyon is almost on shell for small values of the 
B-field the zeroth level approximation for the tachyon potential contributes to $70 \%$ of the expected mass defect. For large values of the B-field with $\operatorname{Pf}(2 \pi B)$ the tachyon potential reduces to that of the D-brane anti-D-brane pair. For large values of the B-field with $\operatorname{Pf}(2 \pi B)$ the zeroth level approximation contributes only to $25 \%$ of the mass defect. Note that in this last case the level truncation method is not very successful at the zeroth level. It would be interesting to know whether the inclusion of higher levels for this case converges to the expected answer.

We see that for the case of large values of the B-field with $\operatorname{Pf}(2 \pi B)>0$ the D0/D4 system reduces to that of the D-brane anti-D-brane system. One is tempted to think that the decoupling of the open string modes in tachyon condensation of the D-brane anti-D-brane system can be understood from the description of the D0/D4 bound state as an instanton in the noncommutative gauge theory of the D4-brane for large values of the B-field.

\section{Acknowledgments}

The author thanks Shinji Hirano, Nissan Itzhaki and especially Shiraz Minwalla for discussions. He is grateful for encouragement from Joe Polchinski and Ashoke Sen. The work of the author is supported by NSF grant PHY97-2202.

\section{A. The twist operator and its correlation function}

This appendix discusses the definition of the bosonic and fermionic twist operators and calculates their correlations functions. The twist operators are located at the boundary of the world sheet. Thus they are on the real axis when the world sheet is on the upper half plane. The bosonic twist operator $\sigma_{\nu}$ inserted at the origin changes the boundary conditions of the open string as shown in the Figure 1. The insertion of $\sigma_{\nu}$ changes the boundary conditions of the string to that of a string joining the D0brane to the D4-brane. The anti-twist operator $\sigma_{-\nu}$ changes the boundary conditions to that of a string joining the D4-brane to the D0-brane. From the mode expansions in (2.4) and (2.7) the bosonic twist operators $\sigma_{\nu}$ and $\sigma_{-\nu}$ have the following OPE with the world sheet bosons

D4-brane
0

Figure 1: Insertion of $\sigma_{\nu}$ at the origin changes the boundary condition to that of a string joining the D0-brane and the D4-brane.

$$
\partial X^{+}(z) \sigma_{\nu}(w)=\frac{1}{(z-w)^{(1-\nu)}} \tau_{\nu}(w) \quad \partial X^{-}(z) \sigma_{\nu}(w)=\frac{1}{(z-w)^{\nu}} \tau_{\nu}^{\prime}(w)
$$




$$
\begin{array}{cc}
\partial X^{+}(z) \sigma_{-\nu}(w)=\frac{1}{(z-w)^{\nu}} \tau_{-\nu}(w) & \partial X^{-}(z) \sigma_{-\nu}(w)=\frac{1}{(z-w)^{(1-\nu)}} \tau_{-\nu}^{\prime}(w) \\
\bar{\partial} X^{+}(\bar{z}) \sigma_{\nu}(w)=-\frac{1}{(\bar{z}-w)^{1-\nu}} \tau_{\nu}(w) & \bar{\partial} X^{-}(\bar{z}) \sigma_{\nu}(w)=-\frac{1}{(\bar{z}-w)^{\nu}} \tau_{\nu}^{\prime}(w) \\
\bar{\partial} X^{+}(\bar{z}) \sigma_{-\nu}(w)=-\frac{1}{(\bar{z}-w)^{\nu}} \tau_{-\nu}(w) & \bar{\partial} X^{-}(\bar{z}) \sigma_{-\nu}(w)=-\frac{1}{(\bar{z}-w)^{(1-\nu)}} \tau_{-\nu}^{\prime}(w)
\end{array}
$$

$w$ is a point on the real axis. $\tau$ 's are the excited twist operators. We are interested in obtaining the following four point function of the twist fields

$$
\begin{aligned}
& Z_{1}\left(z_{1}, z_{2}, z_{3}, z_{4}\right)=\left\langle\sigma_{-\nu}\left(z_{1}\right) \sigma_{\nu}\left(z_{2}\right) \sigma_{-\nu}\left(z_{3}\right) \sigma_{\nu}\left(z_{4}\right)\right\rangle \\
& Z_{2}\left(z_{1}, z_{2}, z_{3}, z_{4}\right)=\left\langle\sigma_{1-\nu}\left(z_{1}\right) \sigma_{-(1-\nu}\left(z_{2}\right) \sigma_{\nu}\left(z_{3}\right) \sigma_{-\nu}\left(z_{4}\right)\right\rangle
\end{aligned}
$$

where $z_{1}, z_{2}, z_{3}, z_{4}$ are four points on the real axis. We will demonstrate the calculation for $Z_{1}$, the calculation of $Z_{2}$ follows along similar lines. We follow the method in [27] and as developed for open strings in [20]. Consider the auxiliary correlators

$$
g(z, w)=\frac{\left\langle-\frac{1}{2} \partial X^{+}(z) \partial X^{-}(w) \sigma_{-\nu}\left(z_{1}\right) \sigma_{\nu}\left(z_{2}\right) \sigma_{-\nu}\left(z_{3}\right) \sigma_{\nu}\left(z_{4}\right)\right\rangle}{\left\langle\sigma_{-\nu}\left(z_{1}\right) \sigma_{\nu}\left(z_{2}\right) \sigma_{-\nu}\left(z_{3}\right) \sigma_{\nu}\left(z_{4}\right)\right\rangle}
$$

and

$$
h(\bar{z}, w)=\frac{\left\langle-\frac{1}{2} \bar{\partial} X^{+}(\bar{z}) \partial X^{-}(w) \sigma_{-\nu}\left(z_{1}\right) \sigma_{\nu}\left(z_{2}\right) \sigma_{-\nu\left(z_{3}\right)} \sigma_{\nu}\left(z_{4}\right)\right\rangle}{\left\langle\sigma_{-\nu}\left(z_{1}\right) \sigma_{\nu}\left(z_{2}\right) \sigma_{-\nu\left(z_{3}\right)} \sigma_{\nu}\left(z_{4}\right)\right\rangle}
$$

Define

$$
\omega_{\nu}(z)=\frac{1}{\left[\left(z-z_{1}\right)\left(z-z_{3}\right)\right]^{\nu}} \frac{1}{\left[\left(z-z_{2}\right)\left(z-z_{4}\right)\right]^{1-\nu}}
$$

Now $g(z, w)$ is given by

$$
\begin{aligned}
g(z, w) & =\omega_{\nu}(z) \omega_{1-\nu}(w)\left(\nu \frac{\left(z-z_{1}\right)\left(z-z_{3}\right)\left(w-z_{2}\right)\left(w-z_{4}\right)}{(z-w)^{2}}\right. \\
& \left.+(1-\nu) \frac{\left(z-z_{2}\right)\left(z-z_{4}\right)\left(w-z_{1}\right)\left(w-z_{3}\right)}{(z-w)^{2}}+A\left(z_{1}, z_{2}, z_{3}, z_{4}\right)\right)
\end{aligned}
$$

The form for $g(z, w)$ given above has the required singularity structure so that the (A.1) is obeyed. When $z \rightarrow w$, then

$$
g(z, w)=\frac{1}{(z-w)^{2}}
$$

$h(\bar{z}, w)$ is given by

$$
\begin{aligned}
h(\bar{z}, w) & =-\omega_{\nu}(\bar{z}) \omega_{1-\nu}(w)\left(\nu \frac{\left(\bar{z}-z_{1}\right)\left(\bar{z}-z_{3}\right)\left(w-z_{2}\right)\left(w-z_{4}\right)}{(z-w)^{2}}\right. \\
& \left.+(1-\nu) \frac{\left(\bar{z}-z_{2}\right)\left(\bar{z}-z_{4}\right)\left(w-z_{1}\right)\left(w-z_{3}\right)}{(z-w)^{2}}+A\left(z_{1}, z_{2}, z_{3}, z_{4}\right)\right)
\end{aligned}
$$


This form for $h(\bar{z}, w)$ also has the singularity structure so that the (A.1) is obeyed. It satisfies the condition

$$
h(\bar{z}, w)=-g(\bar{z}, w)
$$

The origin of this condition can be seen from the mode expansions (2.4) and (2.7). The anti-holomorphic components $\bar{\partial} X^{ \pm}(z)$ can be obtained from the holomorphic components by replacing $z$ by $\bar{z}$ along with a negative sign. We now determine $A$ from the monodromy conditions. We have the monodromy condition ${ }^{2}$

$$
\int_{C} g(z, w) d z+\int_{C} h(\bar{z}, w) d \bar{z}=0
$$

Where the contour $C$ is shown in Figure 2. This monodromy condition arises because integration along the contour $C$ gives the change in $X^{+}$for a string ending on the D0-brane at the two ends. There are two equivalent nontrivial contours $C$ and $C^{\prime}$. To solve for $A$ first divide (A.10) by $\omega_{1-\nu}(w)$ and take $w \rightarrow \infty$. We then use the

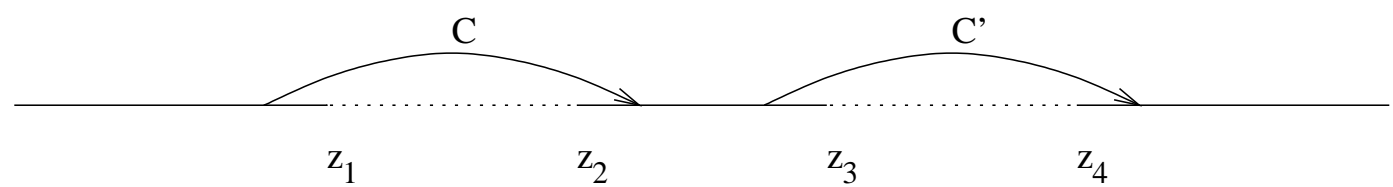

Figure 2: Contours $C$ and $C^{\prime}$ are equivalent nontrivial monodromy contours

$S L(2, R)$ invariance to choose $z_{1}=0, z_{2}=x, z_{3}=1$ and $z_{4}=\infty$, where $0<x<1$. Then $A$ is given by

$$
A=-z_{4} x(1-x) \frac{d}{d x} \ln F(\nu, 1-\nu, 1 ; x)
$$

Here $z_{4}$ stands for $\infty . F(\nu, 1-\nu ; x)$ is the hypergeometric function whose integral representation is given by

$$
F(\nu, 1-\nu, 1 ; x)=\frac{1}{\pi} \sin (\pi \nu) \int_{0}^{1} d y \frac{1}{y^{\nu}(1-y)^{1-\nu}(1-x y)^{\nu}}
$$

Now we can calculate the correlation function $Z_{1}\left(z_{1}, z_{2}, z_{3}, z_{4}\right)$. Consider the following correlation function

$$
\begin{aligned}
\frac{\left\langle T(z) \sigma_{-\nu}\left(z_{1}\right) \sigma_{\nu}\left(z_{2}\right) \sigma_{-\nu}\left(z_{3}\right) \sigma_{\nu}\left(z_{4}\right)\right\rangle}{\left\langle\sigma_{-\nu}\left(z_{1}\right) \sigma_{\nu}\left(z_{2}\right) \sigma_{-\nu}\left(z_{3}\right) \sigma_{\nu}\left(z_{4}\right)\right\rangle} & =\lim _{w \rightarrow z}\left[g(z, w)-\frac{1}{(z-w)^{2}}\right] \\
& =\frac{1}{2} \nu(1-\nu)\left(\frac{1}{\left(z-z_{1}\right)^{2}}+\frac{1}{\left(z-z_{3}\right)^{2}}\right. \\
& \left.-\frac{1}{\left(z-z_{3}\right)^{2}}-\frac{1}{\left(z-z_{4}\right)^{2}}\right) \\
& +\frac{A}{\left(z-z_{1}\right)\left(z-z_{2}\right)\left(z-z_{3}\right)\left(z-z_{4}\right)}
\end{aligned}
$$

\footnotetext{
${ }^{2}$ We are neglecting instanton sectors as the D4-brane is extended in the spatial directions.
} 
where $T$ is the stress energy tensor. From the operator product

$$
T(z) \sigma_{\nu}\left(z_{2}\right)=\frac{1}{2} \frac{\nu(1-\nu) T\left(z_{2}\right)}{\left(z-z_{2}\right)^{2}}+\frac{\partial_{z_{2}} \sigma_{\nu}\left(z_{2}\right)}{z-z_{2}}
$$

we can obtain the following differential equation for $Z_{1}\left(z_{1}, z_{2}, z_{3}, z_{4}\right)$

$$
\begin{aligned}
\partial_{z_{2}} \ln Z_{1}\left(z_{1}, z_{2}, z_{3}, z_{4}\right) & =-\nu(1-\nu)\left(\frac{1}{\left(z_{2}-z_{1}\right)}+\frac{1}{\left(z_{2}-z_{3}\right)}-\frac{1}{\left(z_{3}-z_{4}\right)}\right) \\
& +\frac{A}{\left(z_{2}-z_{1}\right)\left(z_{2}-z_{3}\right)\left(z_{2}-z_{4}\right)}
\end{aligned}
$$

Substituting the values of $z_{1}, z_{2}, z_{3}, z_{4}$ in the above equation we obtain

$$
\partial_{x} Z_{1}(x)=-\nu(1-\nu)\left(\frac{1}{x}-\frac{1}{1-x}\right)-\frac{d}{d x} \ln F(\nu, 1-\nu, 1 ; x)
$$

This equation can easily be integrated to obtain

$$
Z_{1}(x)=\frac{1}{[x(1-x)]^{\nu(1-\nu)} F(\nu, 1-\nu, 1, x)}
$$

We now use the $S L(2, R)$ invariance to obtain $Z_{1}\left(z_{1}, z_{2}, z_{3}, z_{4}\right)$. We get

$$
Z_{1}\left(z_{1}, z_{2}, z_{3}, z_{4}\right)=z_{21}^{-2 h} z_{31}^{2 h} z_{41}^{-2 h} z_{32}^{-2 h} z_{42}^{2 h} z_{43}^{-2 h} \frac{1}{F(\nu, 1-\nu, 1 ; x)}
$$

where $h=\nu(1-\nu) / 2, z_{i j}=z_{i}-z_{j}$ and

$$
x=\frac{z_{21} z_{43}}{z_{31} z_{42}}
$$

To construct the fermionic twist operators we first bosonize the fermions by

$$
\psi^{+}(w)=e^{i H(w)} \quad \psi^{-}(w)=e^{-i H(w)}
$$

where $H$ is a free boson. The fermionic twist operator are is given by $e^{i \nu H}$ and the anti-twist operator is given by $e^{-i \nu H}$. The correlation function for the fermionic twists are easy to evaluate and they are given by

$$
\left\langle e^{-i \nu H\left(z_{1}\right)} e^{i \nu H\left(z_{2}\right)} e^{-i \nu H\left(z_{3}\right)} e^{-i \nu H\left(z_{4}\right)}\right\rangle=z_{21}^{-\nu^{2}} z_{31}^{\nu^{2}} z_{41}^{-\nu^{2}} z_{32}^{-\nu^{2}} z_{42}^{\nu^{2}} z_{43}^{-\nu^{2}}
$$

Putting the bosonic and the fermionic twists together one obtains

$$
\left\langle\Sigma_{-\nu}\left(z_{1}\right) \Sigma_{\nu}\left(z_{2}\right) \Sigma_{-\nu}\left(z_{3}\right) \Sigma_{\nu}\left(z_{4}\right)\right\rangle=z_{21}^{-\nu} z_{31}^{\nu} z_{41}^{-\nu} z_{32}^{-\nu} z_{42}^{\nu} z_{43}^{-\nu} \frac{1}{F(\nu, 1-\nu, 1, x)}
$$

Using similar methods we obtain the correlation function $Z_{2}\left(z_{1}, z_{2}, z_{3}, z_{4}\right)$. It is given by

$$
Z_{2}\left(z_{1}, z_{2}, z_{3}, z_{4}\right)=z_{21}^{-2 h} z_{31}^{-2 h} z_{41}^{2 h} z_{32}^{2 h} z_{42}^{-2 h} z_{43}^{-2 h} \frac{1}{F(\nu, 1-\nu, 1 ; y)}
$$


Here $y=x /(x-1)$. Using $Z_{2}$ we can find the following correlation functions

$$
\begin{aligned}
\left\langle\Sigma_{1-\nu}\left(z_{1}\right) \Sigma_{-(1-\nu)}\left(z_{2}\right) \Sigma_{\nu}\left(z_{3}\right) \Sigma_{-\nu}\left(z_{4}\right)\right\rangle & =\frac{1}{z_{21}^{1-\nu} z_{43}^{\nu}} \frac{1}{F(\nu, 1-\nu, 1 ; y)} \\
\left\langle\Sigma_{-\nu}\left(z_{1}\right) \Sigma_{\nu}\left(z_{2}\right) \Sigma_{-(1-\nu)}\left(z_{3}\right) \Sigma_{1-\nu}\left(z_{4}\right)\right\rangle & =\frac{1}{z_{21}^{\nu} z_{43}^{1-\nu}} \frac{1}{F(\nu, 1-\nu, 1 ; y)}
\end{aligned}
$$

For completeness we write down the two point function of the twist operators. This is fixed by conformal invariance.

$$
\left\langle\Sigma_{\nu}\left(z_{1}\right) \Sigma_{-\nu}\left(z_{2}\right)\right\rangle=\frac{1}{\left(z_{2}-z_{1}\right)^{\nu}}
$$

We have chosen a normalization for the two point function which differs from the conventional one to make sure all coefficients are real in the tachyon potential. The four point function of the twist operators in (A.22) and (A.24) are consistent with this normalization of the two point function ${ }^{3}$.

\section{B. Details on the calculation of the tachyon potential}

The vertex operators $\hat{T}$ and $\hat{E}$ are all primary, therefore they transform under a conformal transformation $f$ as

$$
f \circ \mathcal{O}(0)=\left(f^{\prime}(0)\right)^{h} \mathcal{O}(0)
$$

Here $\mathcal{O}$ is a primary operator of dimension $h$.

\section{B.1 The quadratic term}

We now focus on the evaluation of the quadratic term in the string field theory action given by

$$
S^{(2)}=\frac{1}{4 g^{2}}\left\langle\left\langle\left(\hat{Q}_{B} \hat{\Phi}\right)\left(\hat{\eta}_{0} \hat{\Phi}\right)\right\rangle\right\rangle
$$

Substituting $\hat{\Phi}=\hat{T}+\hat{E}$, the terms which contribute in $S^{(2)}$ are

$$
S^{2}=\frac{1}{4 g^{2}}\left(\left\langle\left\langle\left(\hat{Q}_{B} \hat{T}\right)\left(\hat{\eta}_{0} \hat{T}\right)\right\rangle\right\rangle+\left\langle\left\langle\left(\hat{Q}_{B} \hat{E}\right)\left(\hat{\eta}_{0} \hat{E}\right)\right\rangle\right\rangle\right)
$$

The cross terms do not contribute due to twist conservation. $\hat{T}$ is an operator of dimension $-\left(1-\left(\nu+\nu^{\prime}\right)\right) / 2$ and $\hat{E}$ is an operator of dimention $\left(1-\left(\nu+\nu^{\prime}\right)\right) / 2$. We calculate the correlations functions on the upper half plane. Using (A.25) and evaluating the traces over the Chan-Paton factors and grouping the terms we obtain

$$
\begin{aligned}
S^{(2)} & =\frac{1}{g^{2}}\left(+\frac{1}{2}\left(1-\left(\nu+\nu^{\prime}\right)\right) z_{21}^{1-\left(\nu+\nu^{\prime}\right)}\left(g_{1}^{(2) \prime}(0) g_{2}^{(2) \prime}(0)\right)^{-\left(1-\left(\nu+\nu^{\prime}\right) / 2\right.} t_{-} t_{+}\right. \\
& \left.-\frac{1}{2}\left(1-\left(\nu+\nu^{\prime}\right)\right) z_{21}^{-1+\left(\nu+\nu^{\prime}\right)}\left(g_{1}^{(2) \prime} g_{2}^{(2) \prime}\right)^{\left(1-\left(\nu+\nu^{\prime}\right) / 2\right.} e_{-} e_{+}\right)
\end{aligned}
$$

\footnotetext{
${ }^{3}$ If we had worked with the conventional normalization we can ensure that the coefficients of the tachyon potential are real by a redefinition of the tachyon field.
} 
where $z_{1}=g_{1}^{(2)}(0)$ and $z_{2}=g_{2}^{(2)}(0)$. The $g$ 's are defined in [6]. We write them down here for completeness

$$
\begin{aligned}
g_{1}^{(2)}=\tan \left(-\frac{\pi}{4}\right) & g_{1}^{(2) \prime}=2 \\
g_{2}^{(2)}=\tan \left(\frac{\pi}{4}\right) & g_{1}^{(2) \prime}=2
\end{aligned}
$$

Substituting the values of each of the terms in $S^{(2)}$ we obtain

$$
S^{(2)}=\frac{1}{g^{2}}\left(\frac{1}{2}\left(1-\left(\nu+\nu^{\prime}\right)\right) t_{-} t_{+}-\frac{1}{2}\left(1-\left(\nu+\nu^{\prime}\right)\right) e_{-} e_{+}\right)
$$

\section{B.2 The cubic term}

The cubic term in the superstring field theory action is given by

$$
S^{(3)}=\frac{1}{12 g^{2}}\left\langle\left\langle\left(\hat{Q}_{B} \hat{\Phi}\right)\left(\hat{\Phi}\left(\hat{\eta}_{0} \hat{\Phi}\right)-\left(\hat{\eta}_{0} \hat{\Phi} \hat{\Phi}\right)\right\rangle\right\rangle\right.
$$

Substituting $\hat{\Phi}=\hat{T}+\hat{E}$ in $S^{(3)}$ we see that it vanishes. This can be easily seen by taking the traces of the external Chan-Paton factors.

\section{B.3 The quartic term}

The quartic term in the string field theory action is given by

$$
S^{(4)}=\frac{1}{48 g^{2}}\left\langle\left\langle\left(\hat{Q}_{B} \hat{\Phi}\right)\left(\hat{\Phi}^{2}\left(\hat{\eta}_{0} \hat{\Phi}\right)-2 \hat{\Phi}\left(\hat{\eta}_{0} \hat{\Phi}\right)+\left(\hat{\eta}_{0} \hat{\Phi}\right) \hat{\Phi}^{2}\right)\right\rangle\right\rangle
$$

From the fact that there should be only three $c$ ghosts for the correlation functions to contribute we can see that after the substitution $\hat{\Phi}=\hat{T}+\hat{E}$ there is a factorization of the action given by

$$
S^{(4)}=\mathcal{S} \times \mathcal{T}
$$

where

$$
\begin{aligned}
\mathcal{S} & =\frac{1}{48 g^{2}}\left\langle\left\langle( Q _ { B } \otimes \sigma _ { 3 } O \otimes \sigma _ { 1 } ) \left(\left(O \otimes \sigma_{1}\right)^{2}\left(\eta_{0} \otimes \sigma_{3} O \otimes \sigma_{1}\right)\right.\right.\right. \\
& \left.\left.\left.-2 O \otimes \sigma_{1}\left(\eta_{0} \otimes \sigma_{3} O \otimes \sigma_{1}\right)+\left(\eta_{0} \otimes \sigma_{3} O \otimes \sigma_{1}\right)\left(O \otimes \sigma_{1}\right)^{2}\right)\right\rangle\right\rangle \\
\mathcal{T} & =\langle\langle P P P P\rangle\rangle
\end{aligned}
$$

Here $O=\xi c e^{-\phi}$ and

$$
\begin{aligned}
P & =t_{+} \Sigma_{\nu} \Sigma_{\nu^{\prime}} \otimes \sigma_{+}+t_{-} \Sigma_{-\nu} \Sigma_{-\nu^{\prime}} \otimes \sigma_{-} \\
& +e_{+} \Sigma_{1-\nu} \Sigma_{1-\nu^{\prime}} \otimes \sigma_{+}+e_{-} \Sigma_{-(1-\nu)} \Sigma_{-\left(1-\nu^{\prime}\right)} \otimes \sigma_{-}
\end{aligned}
$$

The value of $\mathcal{S}$ has been calculated in [5, 6]. $\mathcal{S}=-1 /\left(2 g^{2}\right)$. We have to use the correlation functions found in Appendix A to evaluate $\mathcal{T}$. As an example we write down the coefficient of $\left(t_{-} t_{+}\right)^{2}$

$$
\begin{gathered}
2\left(g_{1}^{(4) \prime}(0) g_{2}^{(4) \prime}(0) g_{3}^{(4) \prime}(0) g_{3}^{(4) \prime}(0)\right)^{\left(\nu+\nu^{\prime}\right) / 2} \\
\left\langle\Sigma_{-\nu}\left(z_{1}\right) \Sigma_{\nu}\left(z_{2}\right) \Sigma_{-\nu}\left(z_{3}\right) \Sigma_{\nu}\left(z_{4}\right)\right\rangle\left\langle\Sigma_{-\nu^{\prime}}\left(z_{1}\right) \Sigma_{\nu^{\prime}}\left(z_{2}\right) \Sigma_{-\nu^{\prime}}\left(z_{3}\right) \Sigma_{\nu^{\prime}}\left(z_{4}\right)\right\rangle
\end{gathered}
$$


where $z_{1}=g_{1}^{(4)}(0) z_{2}=g_{2}^{(4)}(0) z_{3}=g_{3}^{(4)}(0) z_{4}=g_{4}^{(4)}(0)$. and the $g$ 's are defined in [6] They are given by

$$
\begin{aligned}
& g_{1}^{(4)}(z)=-4+6 z-9 z^{2}+\cdots \\
& g_{2}^{(4)}(z)=-1+\frac{3}{4} z-\frac{3}{16} z^{2}+\cdots \\
& g_{3}^{(4)}(z)=0+\frac{2}{3} z+\frac{1}{9} z^{2}+\cdots \\
& g_{4}^{(4)}(z)=2+3 z+3 z^{2}+\cdots
\end{aligned}
$$

Substituting these values in $(\mathbb{B . 1 2})$ and using the correlation function in $(\mathrm{A.22})$ we find that the coefficient is that given in (3.17). Note that the value of argument $x$ of the hypergeometric function is $1 / 2$ and $y=-1$.

\section{References}

[1] V.A. Kostelecky and S. Samuel, "The static tachyon potential in the open bosonic string theory," Phys. Lett. B 207 (1988) 169; "On a nonperturbative vacuum for the open bosonic string," Nucl. Phys. B 336 (1990) 263.

[2] A. Sen and B. Zweibach, "Tachyon condensation in string field theory," J. High Energy Phys. 03 (2000) 002, hep-th/9912249.

[3] W. Taylor, "D-brane effective field theory from string field theory," hep-th/0001201.

[4] N. Moeller and W. Taylor, "Level truncation and the tachyon in open bosonic string field theory," hep-th/0002237.

[5] N. Berkovits, "The tachyon potential in open Neveu-Schwarz string field theory," J. High Energy Phys. 04 (2000) 022, hep-th/0001084.

[6] N. Berkovits, A. Sen and B. Zweibach, "Tachyon condensation in superstring field theory," hep-th/0002211.

[7] P. De Smet and J. Raeymaekers, "Level four approximation to the tachyon potential in superstring field theory," J. High Energy Phys. 05 (2000) 051, hep-th/0003220.

[8] A. Iqbal and A. Naqvi, "Tachyon condensation on a non-BPS D-brane," hep-th/0004015.

[9] L. Rastelli, B. Zwiebach, "Tachyon potentials, star products and universality," hep-th/0006240.

[10] J.A. Harvey and P. Kraus, "D-branes as unstable lumps in bosonic open string field theory," J. High Energy Phys. 04 (2000) 012, hep-th/0002117. 
[11] R. de Mello Koch, A. Jevicki, M. Mihailescu and R. Tartar, "Lumps and p-branes in open string field theory," hep-th/0003031.

[12] N. Moeller, A. Sen and B. Zweibach, "D-branes as tachyon lumps in string field theory," hep-th/0005036.

[13] R. Gopakumar, S. Minwalla and A. Strominger, "Noncommutative solitons," J. High Energy Phys. 05 (2000) 020, hep-th/0003160.

[14] K. Dasgupta, S. Mukhi and R. Govindan, "Noncommutative tachyons," J. High Energy Phys. 06 (2000) 022, hep-th/005006.

[15] J.A. Harvey, P. Kraus, F. Larsen and E. Martinec, "D-branes and strings as noncommutative solitons," hep-th/0005031.

[16] D. P. Jatkar, G. Mandal and S.R. Wadia, "Nielsen-Olesen vortices in noncommutative Abelian Higgs model," hep-th/0007078.

[17] R. Gopakumar, S. Minwalla and A. Strominger, "Symmetry restoration and tachyon condensation in open string theory," hep-th/0007226.

[18] E. Witten, "Noncommutative tachyons and string field theory," hep-th/0007071.

[19] A. Sen, "Universality of the tachyon potential," J. High Energy Phys. 12 (1999) 027, hep-th/9911116.

[20] E. Gava, K.S. Narain and M.H. Sarmardi, "On the bound states of p-and (p+2)branes," Nucl. Phys. B 504 (1997) 214, hep-th/9704006.

[21] N. Berkovits, "Super-Poincare invariant superstring field theory," Nucl. Phys. B 450 (1995) 90, hep-th//9503099; "A new approach to superstring field theory," proceedings to the $32^{\text {nd }}$ International Symposium Ahrenshoop on the theory of elementary particles, Fortschritte der Physik (Progress of Physics), 48 (2000) 31, hep-th/9912121.

[22] N. Seiberg and E. Witten, "String theory and noncommutative geometry," J. High Energy Phys. 09 (1999) 032, hep-th/9908142.

[23] M. Abramowitz and I.A. Stegun, Handbook of mathematical functions (Dover publications, 1965).

[24] N.A. Obers and B. Pioline, "U duality and M theory," Phys. Rep. 318 (1999) 113, hep-th/9809039, hep-th/9809039.

[25] A. Dhar, G. Mandal, S.R. Wadia and K.P. Yogendran, "D1/D5 system with B-field, noncommutative geometry and the CFT of the Higgs branch," Nucl. Phys. B 575 (2000) 177, hep-th/9910194.

[26] N. Nekrasov and A. Schwarz, "Instanton on noncommutative $R^{4}$ and $(2,0)$ superconformal six dimensional theory," Comm. Math. Phys. 198 (1998) 689, hep-th/9802068. 
[27] L. Dixon, D. Friedan, E. Martinec and S. Shenker, "The conformal field theory of orbifolds," Nucl. Phys. B 282 (1987) 13. 